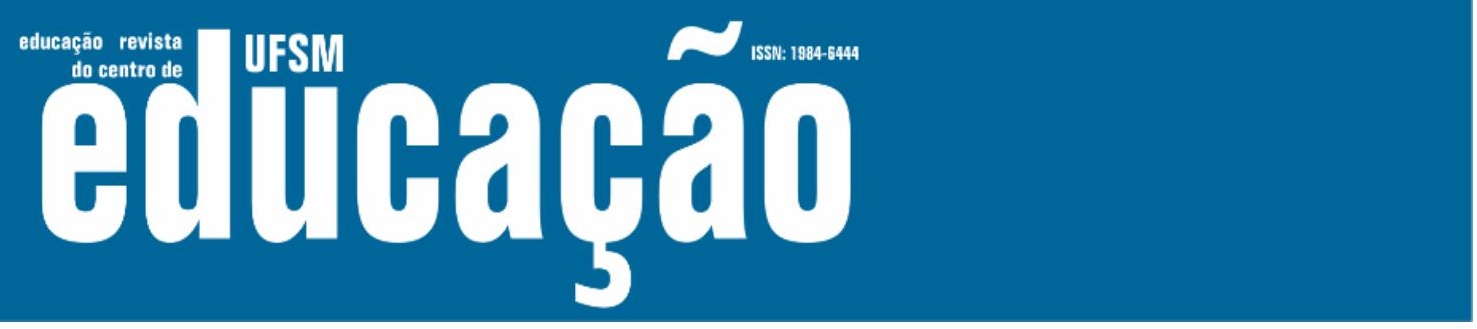

ISSN: 1984-6444 | http://dx.doi.org/10.5902/1984644448156

\title{
Usos de la noción de relación con el saber en la investigación educativa y psicopedagógica
}

Uses of the notion of relationship with knowledge in educational and psychopedagogical research

Usos da noção de rapport au savoir na pesquisa educacional e psicopedagógica

\author{
Soledad Vercellino \\ Universidad Nacional de Río Negro. Centro Interdisciplinario de Estudios sobre Derechos, Inclusión y \\ Sociedad. Río Negro, Argentina \\ svercellino@unrn.edu.ar -https://orcid.org/0000-0002-1087-5455
}

Recebido em 14 de julho de 2020

Aprovado em 19 de outubro de 2020

Publicado em 21 de dezembro de 2020

\section{RESUMO}

Este artículo teórico da cuenta del incipiente, pero vigoroso, desarrollo de la noción de relación con el saber en el ámbito de la investigación educativa, no sólo a nivel internacional, sino también en Argentina. Muestra como esa noción cuenta con la capacidad heurística de articular en el análisis del aprendizaje, las relaciones y múltiples influencias e imbricaciones de las dimensiones destacadas por las teorías del aprendizaje escolar, es decir, puede dar cuenta como el aprender acontece en la encrucijada de diferentes procesos cognitivos y metacognitivos con su anclaje sociohistórico y situacional; el vínculo del sujeto aprendiente con los objetos de aprendizaje; las características epistemológicas de los objetos de aprendizaje y la singularidad del psiquismo de quien aprende.

Interesa particularmente poner en relevancia las investigaciones psicopedagógicas realizadas a nivel local, tanto en el marco de proyectos de investigación como de tesis de grado y posgrado, dando cuenta de las problematizaciones y producción científica que la misma ha permitido generar.

Palavras-chave: Relación con el saber; Aprendizaje escolar; Investigación psicopedagógica. 


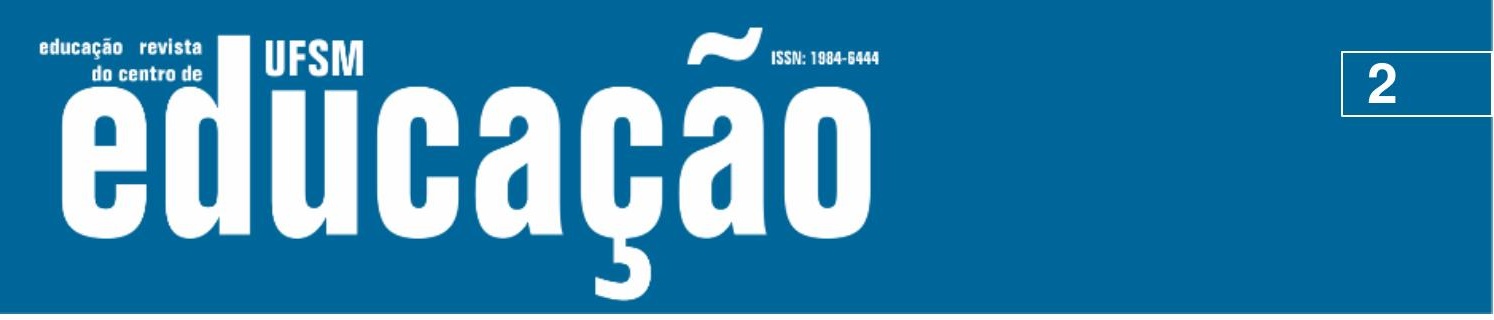

ISSN: 1984-6444 | http://dx.doi.org/10.5902/1984644448156

\section{ABSTRACT}

This theoretical article reports the incipient, but vigorous, development of the notion of relationship with knowledge in the field of educational research, not only at the international level, but also in Argentina. It shows how this notion has the heuristic capacity to articulate, in the analysis of learning, the relationships and multiple influences and overlaps of the dimensions highlighted by theories of school learning, that is, it can account for how learning occurs at the crossroads of different cognitive and metacognitive processes with their sociohistorical and situational anchorage; the link of the learning subject with the learning objects; the epistemological characteristics of learning objects and the uniqueness of the learner's psyche.

It is particularly interesting to highlight the psycho-pedagogical investigations carried out at the local level, both within the framework of research projects and undergraduate and postgraduate theses, accounting for the problems and scientific production that it has allowed to generate.o.

Keywords: Relationship with knowledge; Scholar learning; Psycho-pedagogical research.

\section{RESUMO}

Este artigo teórico relata o incipiente, mas vigoroso, desenvolvimento da noção de rapport au savoir no campo da pesquisa educacional, não apenas no nível internacional, mas também na Argentina. Mostra como essa noção tem capacidade heurística de articular, na análise da aprendizagem, as relações e múltiplas influências e sobreposições das dimensões destacadas pelas teorias da aprendizagem escolar, ou seja, pode explicar como ocorre a aprendizagem na encruzilhada de diferentes processos cognitivos e metacognitivos com sua ancoragem sócio-histórica e situacional; o vínculo da disciplina com os objetos de aprendizagem; as características epistemológicas dos objetos de aprendizagem e a singularidade da psique do aluno.

É particularmente interessante destacar as investigações psicopedagógicas realizadas em nível local, tanto no âmbito de projetos de pesquisa quanto de teses de graduação e pós-graduação, respondendo pelos problemas e produção científica que ela permitiu gerar.

Palavras-chave: Rapport au savoir; Aprendizagem; Investigações psicopedagógicas.

\section{Introducción ${ }^{1}$}

Desde hace varios años venimos explorando la fertilidad de analizar el trabajo singular y situado que supone el aprendizaje escolar a partir de los aportes heurísticos de las teorías que se ocupan de estudiar la relación con el saber (RcS). Esta noción, 


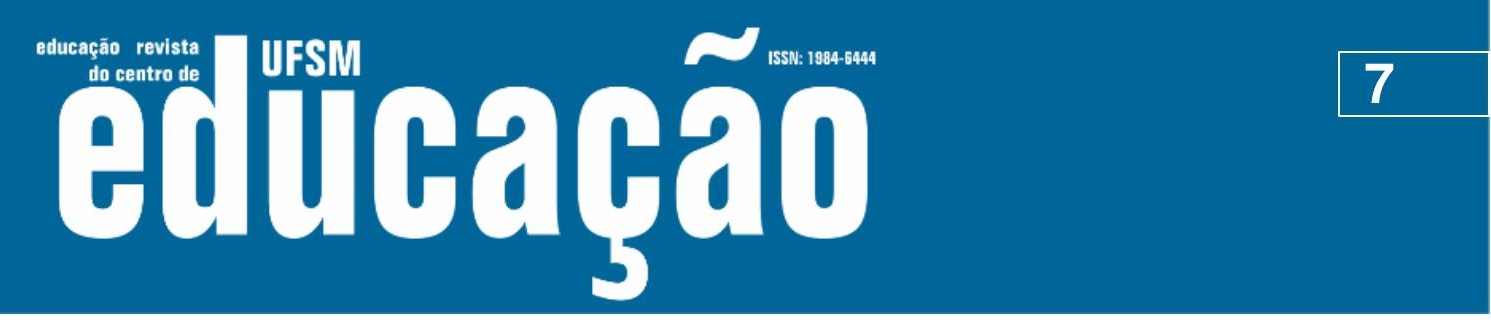

ISSN: 1984-6444 | http://dx.doi.org/10.5902/1984644448156

Tabla 1 - Grupos de investigación que refieren a la relación con el saber en Argentina.

\begin{tabular}{|c|c|c|c|}
\hline Identificación & Inscripción institucional & Principales trabajos & Foco de interés \\
\hline $\begin{array}{l}\text { Grupo } \\
\text { Patagonia } \\
\text { Norte }\end{array}$ & $\begin{array}{l}\text { Centro Interdisciplinario de } \\
\text { Estudios sobre Inclusión y } \\
\text { Sociedad (CIEDIS) } \\
\text { Universidad Nacional de } \\
\text { Río Negro. } \\
\text { Centro Regional Zona } \\
\text { Atlántica (CURZA) } \\
\text { Universidad Nacional del } \\
\text { Comahue }\end{array}$ & $\begin{array}{c}\text { LA AUTORA et al, } \\
\text { 2018. } \\
\text { LA AUTORA, 2015 } \\
\text { LA AUTORA, Van } \\
\text { Den Heuvel y } \\
\text { Guerreiro, 2014; LA } \\
\text { AUTORA, 2014 } \\
\text { van den Heuvel, R. y } \\
\text { Vercelino, S. (2015) } \\
\text { van den Heuvel (2016 } \\
\text { Andrade, 2016; } \\
\text { Guerreiro 2016 } \\
\text { Tarruella, 2018 } \\
\text { Cardinale, 2018 }\end{array}$ & $\begin{array}{l}\text { Fertilidad teórica de la } \\
\text { noción para la } \\
\text { investigación de los } \\
\text { aprendizajes escolares. } \\
\text { Relación con el saber de } \\
\text { alumnos que finalizan la } \\
\text { educación primaria. } \\
\text { Relación con el saber de } \\
\text { estudiantes que ingresan } \\
\text { a la universidad. }\end{array}$ \\
\hline $\begin{array}{c}\text { Grupo } \\
\text { Córdoba I }\end{array}$ & $\begin{array}{c}\text { Grupo del Centro de } \\
\text { Estudios Avanzados } \\
\text { Universidad Nacional de } \\
\text { Córdoba } \\
\end{array}$ & $\begin{array}{c}\text { Arcanio, Falavigna Y } \\
\text { Soler, } 2013 \\
\text { Falavigna y Arcanio, } \\
2011 \\
\end{array}$ & $\begin{array}{c}\text { Relación con el saber de } \\
\text { estudiantes que ingresan } \\
\text { a la universidad }\end{array}$ \\
\hline $\begin{array}{c}\text { Grupo de } \\
\text { Didáctica de } \\
\text { la } \\
\text { Matemática }\end{array}$ & $\begin{array}{l}\text { Instituto de Investigaciones } \\
\text { en Humanidades y Ciencias } \\
\text { Sociales- Universidad } \\
\text { Nacional de La Plata }\end{array}$ & $\begin{array}{l}\text { Broitman, 2012a y } \\
\text { 2012b; Broitman y } \\
\text { Charlot, 2014. }\end{array}$ & $\begin{array}{l}\text { Relación con los saberes } \\
\text { matemáticos, en particular } \\
\text { en la educación de } \\
\text { adultos que inician su } \\
\text { escolaridad primaria }\end{array}$ \\
\hline $\begin{array}{l}\text { Grupo } \\
\text { Córdoba- } \\
\text { Escuela } \\
\text { secundaria }\end{array}$ & $\begin{array}{l}\text { Facultad de Filosofía y } \\
\text { Humanidades. Universidad } \\
\text { Nacional de Córdoba }\end{array}$ & $\begin{array}{l}\text { Gutiérrez y Uanini, } \\
2015\end{array}$ & $\begin{array}{l}\text { Relación con el saber y } \\
\text { escuela secundaria, con } \\
\text { énfasis en las políticas } \\
\text { educativas y curriculares } \\
\text { (Gutiérrez y Uanini, 2015). }\end{array}$ \\
\hline Grupo CEM & $\begin{array}{c}\text { Centro de Estudios } \\
\text { Multidisciplinarios (CEM). }\end{array}$ & $\begin{array}{c}\text { Diker, 2007; Frigerio y } \\
\text { Diker, } 2005\end{array}$ & $\begin{array}{c}\text { Condiciones de } \\
\text { enseñanza que posibilitan } \\
\text { otras relaciones con el } \\
\text { saber. Análisis de } \\
\text { contextos no escolares }\end{array}$ \\
\hline $\begin{array}{l}\text { Estudios } \\
\text { sobre la } \\
\text { relación con } \\
\text { el saber del } \\
\text { docente }\end{array}$ & $\begin{array}{c}\text { Facultad de Filosofía de la } \\
\text { Universidad de Buenos } \\
\text { Aires. } \\
\text { Facultad de Filosofía y } \\
\text { Humanidades de la } \\
\text { Universidad Nacional de } \\
\text { Córdoba } \\
\text { Universidad Nacional del } \\
\text { Sur. }\end{array}$ & $\begin{array}{c}\text { Tenaglia, 2011; } \\
\text { Ardiles y Borioli, 2010; } \\
\text { Jure, 2010; Monetti, } \\
2003\end{array}$ & $\begin{array}{l}\text { Relación con el saber } \\
\text { disciplinar y con el saber } \\
\text { didáctico }\end{array}$ \\
\hline
\end{tabular}

Fuente: Vercellino (2018, p. 16)

En la región de la Patagonia Norte, desde el 2013 venimos desarrollando estudios sobre la relación con el saber, en el marco de tres proyectos de investigación. El primero ha indagado la fertilidad teórica de la noción para la comprensión 


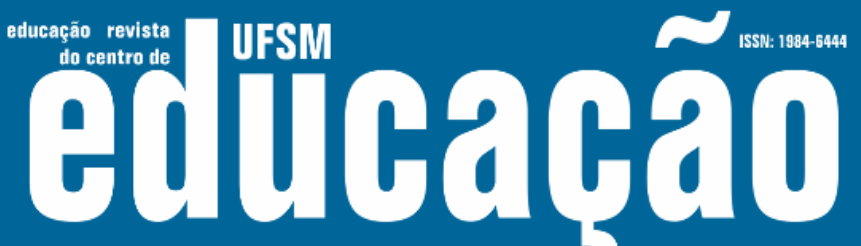

ISSN: 1984-6444 | http://dx.doi.org/10.5902/1984644448156

respectivamente. Valiéndose de los postulados de diferentes corrientes psicoanalíticas, enfatizan en los aspectos no conscientes presentes en la relación con el saber.

Más allá de su inscripción en una u otra escuela, las investigaciones coinciden en que la relación de un sujeto con el saber refiere a los procesos de apropiación y recreación de ciertas prácticas sociales de saberes con sus lógicas de producción, validación y transmisión (CHEVALLARD, 2003) que permiten dar sentido, otorgar significados (CHARLOT, 2008), pensar (BEILLEROT, 1996, p. 112) al mundo y actuar sobre él (BEILLEROT et al., 1998; CHARLOT; 2008).

Ya en los textos lacaneanos que dan origen al concepto de relación con el saber (LACAN, 1965; 2013a), como en los esfuerzos de precisar conceptualmente la noción por parte de autores como Beillerot (1998), Charlot (2008) o Chevallard (2003), queda establecido que distintas prácticas sociales instituyen diferentes modalidades de relación con el saber. No obstante ello, los estudios sobre la 'relación con el saber' abordan escasamente la forma en que una configuración particular de prácticas, como las escolares, performa las relaciones de los alumnos con el saber (VERCELLINO, 2014).

\section{Estrategias metodológicas en los estudios de la relación con el saber}

Los estudios sobre la relación con el saber, ofrecen multiplicidad de estrategias y técnicas de recolección de datos. En la Tabla $N^{\circ} 2$, se presenta una sistematización de las principales estrategias de recolección de datos a las que recurren investigaciones empíricas del campo de los estudios de la "relación con el saber", indicando para cada caso el marco conceptual y metodológico que sirve de referencia. 


\section{工 \\ Futbap̧a

ISSN: 1984-6444 | http://dx.doi.org/10.5902/1984644448156

Tabla 2 - Sistematización de las estrategias de recolección de datos de investigaciones empíricas sobre relación con el saber

\begin{tabular}{|c|c|c|}
\hline INVESTIGACIONES & $\begin{array}{l}\text { MARCO CONCEPTUAL } \\
\text { DE REFERENCIA }\end{array}$ & $\begin{array}{l}\text { ESTRATEGIAS DE RECOLECCIÓN } Y \\
\text { ANÁLISIS DE DATOS }\end{array}$ \\
\hline Allain, J. (2011) & ESCOL & Encuestas a padres. \\
\hline $\begin{array}{l}\text { Ardiles, M, y Borioli, G. } \\
(2010) .\end{array}$ & ESCOL & Relatos autobiográficos. \\
\hline $\begin{array}{c}\text { Arruda,S. y Ueno, M. } \\
(2005)\end{array}$ & ESCOL & Entrevistas, cuestionarios. \\
\hline $\begin{array}{l}\text { Beaucher, C. } \\
\text { Beaucher, V. y Moreau, } \\
\text { D. (2013) }\end{array}$ & ESCOL & Balances de saber \\
\hline Da Silva,V.A. 2008 & Charlot (2005) & Encuestas \\
\hline $\begin{array}{l}\text { de Freitas, E; Bicalho, } \\
\text { M; de Souza, M y Netto, } \\
\text { C (2012) }\end{array}$ & Charlot (2009) & $\begin{array}{c}\text { Balance de saber implementado vía internet } \\
\text { adaptado a la temática de investigación } \\
\text { (saber sobre TIC) }\end{array}$ \\
\hline $\begin{array}{l}\text { Diker, G. (2007) y } \\
\text { Frigerio, G. y Diker, G. } \\
\text { (2005) }\end{array}$ & CREF- ESCOL & $\begin{array}{c}\text { Investigación acción (implementación de } \\
\text { Talleres de producción cultural) y } \\
\text { entrevistas. }\end{array}$ \\
\hline $\begin{array}{l}\text { Falavigna, C. y Arcanio, } \\
\text { M. (2011); Arcanio, M., } \\
\text { Falavigna, C., y Soler, } \\
\text { P. (2013). }\end{array}$ & ESCOL - CREF & $\begin{array}{c}\text { Grupos de discusión y talleres, encuestas, } \\
\text { observaciones }\end{array}$ \\
\hline Grieco, L. (2013) & CREF & $\begin{array}{c}\text { Enfoque clínico observación de grupo, } \\
\text { entrevistas semiestructuradas y técnicas } \\
\text { proyectivas }\end{array}$ \\
\hline Hatchuel,F. (2009) & CREF & $\begin{array}{l}\text { Enfoque clínico. Entrevista a dos } \\
\text { adolescentes. Observación etnográfica de la } \\
\text { clase }\end{array}$ \\
\hline $\begin{array}{c}\text { Hernández y Tort } \\
\text { (2009), Hernández, } \\
\text { (2011) y Hernández, F., } \\
\text { y Padilla-Petry, P. } \\
\text { (2011) }\end{array}$ & ESCOL & Biografía- entrevista en profundidad \\
\hline Jure, E. (2010) & ESCOL & $\begin{array}{c}\text { Investigación biográfico-narrativa a través } \\
\text { de entrevistas semi-estructuradas. }\end{array}$ \\
\hline $\begin{array}{c}\text { Klein, A. Arruda, S. } \\
\text { Passos, M. y Zapparoli, } \\
\text { F. (2010) }\end{array}$ & ESCOL & Observaciones. Entrevistas breves \\
\hline Mairone, C. (2007) & ESCOL & $\begin{array}{l}\text { Análisis documental (leyes, programas), } \\
\text { encuesta. }\end{array}$ \\
\hline Miled, A. (2012) & CREF & Entrevistas en profundidad \\
\hline Monetti, E. (2003) & ESCOL- CREF & Entrevista en profundidad semiestructurada. \\
\hline $\begin{array}{l}\text { Musard, M. y Poggi, M. } \\
\text { (2012) }\end{array}$ & ESCOL & Entrevista flash \\
\hline $\begin{array}{c}\text { Quilaqueo, D. y } \\
\text { Quintriqueo, S. (2010) }\end{array}$ & ESCOL- CREF & Entrevista semiestructurada. \\
\hline $\begin{array}{c}\text { Quintriqueo, S. y Torres, } \\
\text { H. (2012). }\end{array}$ & ESCOL- CREF & Encuestas; entrevistas individuales \\
\hline $\begin{array}{l}\text { Silva, A. y Almeida, M. } \\
\text { (2015) }\end{array}$ & ESCOL & $\begin{array}{l}\text { Análisis de Discurso con actividades de } \\
\text { lectura y preguntas luego. }\end{array}$ \\
\hline
\end{tabular}




\section{تilfoarẫ}

ISSN: 1984-6444 | http://dx.doi.org/10.5902/1984644448156

Adicionalmente, los estudios de la relación con el saber han permitido desarrollar - con diferente profundidad y fundamentación- diferentes metodologías de investigación, como por el ejemplo, el denominado 'método clínico' en los estudios psicoanalíticos o los 'balances de saber' en la propuesta sociantropológica; metodologías que se vinculan a las diferentes dimensiones de análisis enfatizadas: procesos libidinales vinculados al aprender, tipo de actividades intelectuales comprometidas en ese proceso; sentidos adjudicados al saber; formas institucionales de organización y presentación de los saberes, por citar algunas.

La noción de "relación con el saber" es amplia, multirreferencial, en formación, vaga, de contornos y condición inciertos, que está todavía y siempre sobre cantera abierta. En este inacabado de la noción reside su ventaja y su inconveniente: es flexible, amplia y generosa, se puede adaptar al estudio de objetos variados, entonces permanece estrechamente relacionada con el contexto y los objetivos de la investigación en la que está sujeta, y su puesta en práctica variará de acuerdo si el investigador hace hincapié en el aspecto subjetivo de la relación con el saber o sus raíces en múltiples situaciones socioculturales.

No obstante estas diferencias y algunas controversias acontecidas entre referentes de estas escuelas, proponemos y apostamos a la potencia del dialogo entre esas producciones. El carácter abierto, inacabado de la propia noción de 'relación con el saber' es un primer argumento a favor de ese diálogo constructivo. A lo que se suman ciertas coincidencias fundamentales entre las tres escuelas que lo habilitan, entre las que vale destacar: el estar convocadas a producir conocimiento sobre el aprendizaje y sus vicisitudes y su inscripción en un espacio intelectual común, la denominada "filosofía francesa contemporánea" (BADIOU, 2013).

En nuestras investigaciones hemos procurado dimensionalizarla para abordar el aprendizaje escolar en tanto trabajo psíquico, social, singular y situado. La explicitación de ese trabajo de dimensionalización amerita una comunicación específica. 


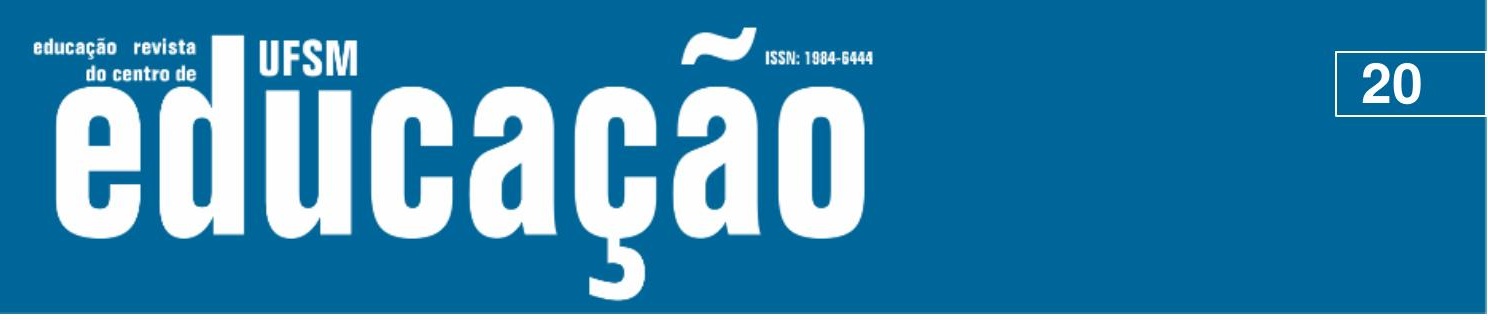

ISSN: 1984-6444 | http://dx.doi.org/10.5902/1984644448156

\section{Referencias}

ARCANIO, Mariana; FALAVIGNA, Carla; SOLER, Paula. Ingreso y desconcierto:¿ nuevas preguntas y viejas estrategias? Sobre los jóvenes, la relación con el conocimiento y la construcción de subjetividades. Cuadernos de Educación, vol. 11, no 11, 2013.

ARDILES, Martha; BORIOLI, Gloria. Las huellas de la academia en los profesores de profesores. Algunos resultados del trabajo de campo. Praxis Educativa, vol. 14, no 14, p. 57-65, 2012.

ARRUDA, Sergio de Mello; UENO, Michele Hidemi. Sobre o ingresso, desistência e permanência no curso de Física da Universidade Estadual de Londrina: algumas reflexões. Ciência \& Educação (Bauru), 2003, vol. 9, no 2, p. 159-175. 2003.

BACCON, Ana Lúcia Pereira; ARRUDA, Sergio de Mello. Estilos de gestão da sala de aula: uma análise a partir da ação docente. Doi: 10.5212/PraxEduc. v. 10i2. 0010. Práxis Educativa, 10(2), 463-487. 2015.

BACCON, Ana Lúcia Pereira; ARRUDA, Sergio de Mello. Teacher knowledge in initial teacher education: elaborating meanings for supervised teaching practice activities.

Ciência \& Educação (Bauru), vol. 16, no 3, p. 507-524. 2010.

BADIOU, Alain. La aventura de La filosofía francesa: a partir de 1960. Eterna Cadência, 2013.

BEAUCHER, Chantal; BEAUCHER, Vincent; MOREAU, Daniel. Contribution à l'opérationnalisation du concept de rapport au savoir. Revue Esprit critique, vol. 17, p. 6-29, 2013.

BEILLEROT, Jacky; LAVILLE, Claudine Blanchard; MOSCONI, Nicole. Saber y relación con el saber. Paidós, 1998.

BETTI, Mauro et al. Os saberes da Educação Física na perspectiva de alunos do Ensino Fundamental: o que aprendem e o que gostariam de aprender. Revista Brasileira de Educação Física Escolar, vol. 1, no 1, p. 155-16. 2015.

BION, Wilfred. Volviendo a pensar. Buenos Aires, Hormé, 1970.

BLANCHARD-LAVILLE, Claudine, et al. L'approche clinique d'inspiration psychanalytique en sciences de l'éducation (note de synthèse). Revue française de Pédagogie, vol. 151, p. 111-162. 2005.

BLANCHARD-LAVILLE, Claudine. Accompagnement clinique et capacité négative. Cahiers de psychologie clinique, no 2, p. 63-80, 2013.

BOURDIEU, Pierre; PASSERON, Jean-Claude. La reproduction. París: Les Editions de Minuit. 1970. 


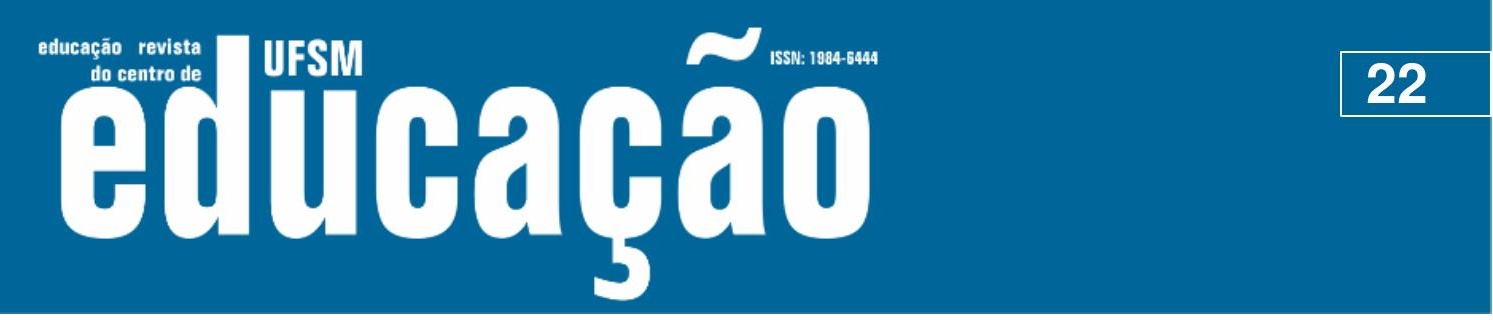

ISSN: 1984-6444 | http://dx.doi.org/10.5902/1984644448156

GAGNON, Mathieu. Penser la question des rapports aux savoirs en éducation: clarification et besoin de recherches conceptuelles. En Les ateliers de l'éthique/The Ethics Forum. Centre de recherche en éthique de l'Université de Montréal. p. 30-42. 2011.

GRIECO, Luis. La dimensión grupal de la Relación con el Saber. Estudio de un caso en el escenario de la Educación Superior. Querencia, no 14, p. 72-99. 2013.

GUERREIRO, Mariana. La relación de sujetos adultos con el saber corporal en el marco de la enseñanza y el aprendizaje del método Pilates (Tesis de pregrado). Universidad Nacional del Comahue, Centro Universitario Regional Zona Atlántica, Viedma. 2016.

GUIRAO-GORIS, Josep Adolf; OLMEDO SALAS, Angela; FERRER FERRANDIS, Esperanza. El artículo de revisión. Revista Iberoamericana de Enfermeria

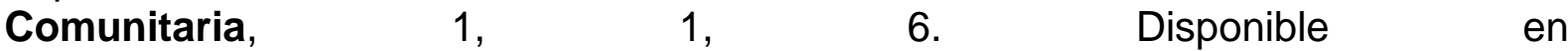
http://revista.enfermeriacomunitaria.org/articuloCompleto.php?ID=7, 2008.

GUTIÉRREZ, Gonzalo; UANINI, Mónica. Transformaciones en los procesos de la escolaridad secundaria argentina (1970-2013). Revista Latinoamericana de Políticas y administración de la Educación, vol. 2, no 2, p. 28-37. 2015.

HATCHUEL, F. La relación de los jóvenes con el saber: entre transmisión e implicaciones inconscientes. Revista de la Facultad de Psicología-Xalapa. Procesos Psicológicos y Sociales. Revista en línea, Universidad de Vera Cruz, México. Recuperado de: http://www.uv.mx/psicologia/files/2013/06/La-relacion-delos-jovenes-con-el-saber.pdf, 2009.

HERNÁNDEZ, Fernando; TORT, Antoni. Cambiar la mirada sobre el fracaso escolar desde la relación de los jóvenes con el saber. Revista Iberoamericana de Educación, vol. 49, no 8, p. 1-11. 2009.

JURE, Elisa. Los docentes noveles y su relación con el saber que enseñan. II Congreso Internacional sobre profesorado principiante e inserción profesional a la docencia. Bs. As, 2010.

KALALI, Fouzia. Rapport au savoir: bilan sur la place du sujet dans les différents travaux. En Symposium «Rapport au savoir: du concept aux usages»-6ème Congrès international d'actualité de la recherche en éducation et en formation, AECSE, Strasbourg, France. 2007.

KLEIN, Alberto Eduardo, et al. Os sentidos da observação astronômica: uma análise com base na relação com o saber. Revista Latino-Americana de Educação em Astronomia, no 10, p. 37-54, 2010.

LACAN, Jaques. "Clase 2. 8 de diciembre de 1965". En: Lacan, J.: Seminario 13. EI objeto del psicoanálisis. 1965. 


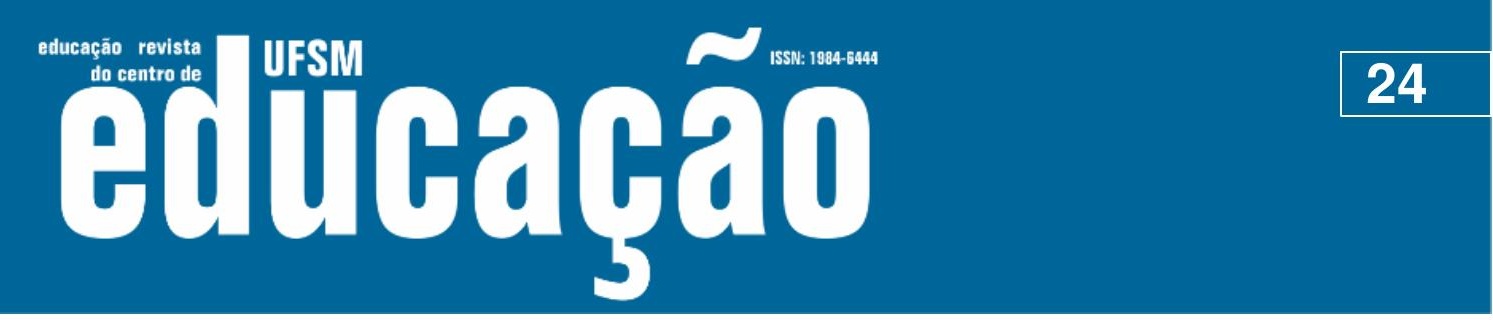

ISSN: 1984-6444 | http://dx.doi.org/10.5902/1984644448156

REIS, Rosimeire. Pesquisas sobre a relação com o saber e com os saberes no Brasil (2000-2013): aspectos preliminares. Revista Eletrônica Científica Ensino Interdisciplinar, vol. 2, no 6. 2016.

RICHARD-BOSSEZ, Ariane. Saisir le rapport aux savoirs en actes à l'école maternelle: Éléments de réflexion conceptuels et empiriques. Rapport au savoir, 17, 123-135. 2013.

ROCHEX, Jean-Yves. The notion of relation to knowledge: convergences and heoretical debates. Educação e Pesquisa, vol. 32, no 3, p. 637-650. 2006.

SILVA, Veleida Anahi da. Relação com o saber na aprendizagem matemática: uma contribuição para a reflexão didática sobre as práticas educativas. Revista Brasileira de Educação, vol. 13, no 37, p. 150-161. 2008.

SOUZA, Maria Celeste Reis Fernandes de. Estudantes em tempo integral no campo: aprendizagens, processos e sentidos. Cadernos de Pesquisa, vol. 46, no 161, p. 756782. 2016.

SOUZA, Maria Celeste Reis Fernandes. Aprendizagens e tempo integral: entre a efetividade e o desejo. Ensaio: Avaliação e Políticas Públicas em Educação, vol. 25, no 95, p. 414-439. 2017.

SOUZA, Maria Celeste Reis Fernandes; CHARLOT, Bernard. Relação com o Saber na Escola em Tempo Integral. Educação \& Realidade, vol. 41, no 4, p. 1071-1093. 2016.

TENAGLIA, Guadalupe. Los rasgos de la relación con el saber del docente en el espacio de formación profesional (taller) en la enseñanza técnica. Estudio clínico. Anuario IICE-Instituto de Investigaciones en Ciencias de la Educación, vol. 2011, p. 419-422. 2011.

THERRIAULT, Geneviève; BADER, Barbara; NDONG ANGOUÉ, C. L'apport de la notion de rapport (s) au (x) savoir (s) en éducation aux sciences et en formation initiale et continue des enseignants du secondaire: des exemples au Québec et au Gabon. Revue Esprit critique, vol. 17, p. 70-94. 2013.

TRÓPIA, Guilherme. A relação epistêmica com o saber de alunos no ensino de biologia por atividades investigativas. Alexandria: Revista de Educação em Ciência e Tecnologia, vol. 8, no 3, p. 55-80. 2015.

TRÓPIA, Guilherme; CALDEIRA, Ademir Donizeti. Vínculos entre a relação com o saber de Bernard Charlot e categorias bachelardianas. Educação, vol. 34, no 3, p. 369-375. 2011.

UNIVERSIDAD NACIONAL DEL COMAHUE. Departamento de Psicopedagogía. Relación de los alumnos con el saber y formato escolar. Investigación teórica y empírica con alumnos de escuelas primarias de la ciudad de Viedma, Río Negro. 2013-2014 (PI V04-084). Viedma, Río Negro. 2013. 


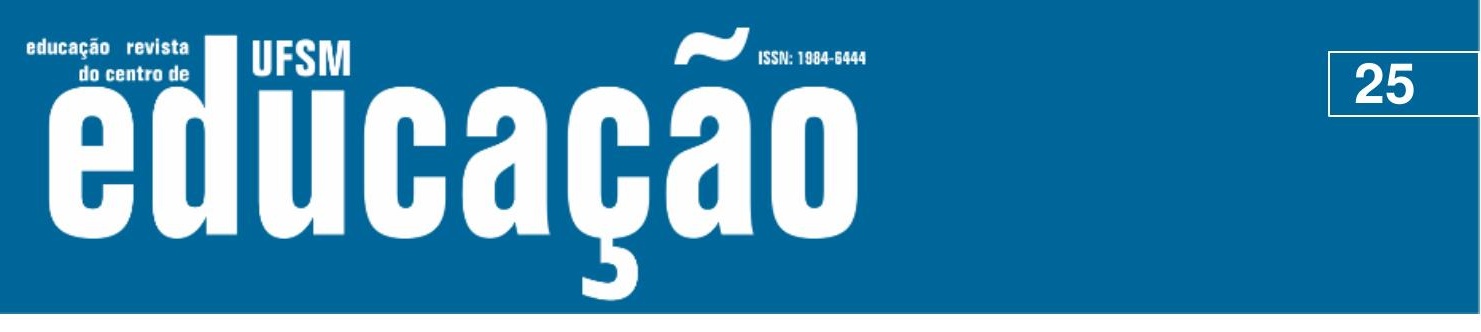

ISSN: 1984-6444 | http://dx.doi.org/10.5902/1984644448156

UNIVERSIDAD NACIONAL DEL COMAHUE. Departamento de Psicopedagogía. La constitución de la relación con el saber en los alumnos del último ciclo del nivel primario y primero del nivel medio. Viedma, Río Negro. 2015-2016 (PI V097). Viedma, Río negro. 2015.

VAN DEN HEUVEL, Romina y LA AUTORA. La movilidad de conceptos en el campo psicopedagógico: Una aproximación a las relaciones entre conceptos propuestos por Jacky Beillerot y Alicia Fernández. Revista Pilquen. Sección Psicopedagogía, 12(1), 1-13. Recuperado de http://www.curza.net/revistapilquen/index.php/Psico/article/view/59, 2015.

VAN DEN HEUVEL, Romina. Movimientos conceptuales en el campo psicopedagógico. Análisis de las relaciones entre conceptos propuestos por Jacky Beillerot y Alicia Fernández (Tesis de pregrado). Universidad Nacional del Comahue, Centro Universitario Regional Zona Atlántica, Viedma. 2016.

VENTURINI, Patrice, et al. Analyse didactique des pratiques d'enseignement de la physique d'une professeure expérimentée. Aster, 2007.

VERCELLINO, Soledad. La 'relación con el saber': Revisitando los comienzos del concepto. Revista Pilquen. Sección Psicopedagogía, 16(11), 1-8.

VERCELLINO, Soledad. Revisión bibliográfica sobre la 'relación con el saber'. Desplazamientos teóricos y posibilidades para el análisis psicopedagógico de los aprendizajes escolares. Revista Electrónica Educare, 19(2), 53-82. doi: https://doi.org/10.15359/ree.19-2.4. 2015.

VERCELLINO, Soledad. Panorama de la investigación sobre relación con el saber en argentina. International Journal Education and Teaching (PDVL) ISSN 2595-2498, 1(3), 96-112. DOI: https://doi.org/10.31692/2595-2498.v1i3.64, 2018.

VERCELLINO, Soledad., VAN DEN HEUVEL, Romina, GUERREIRO, Mariana. Deslocamentos teóricos da noção da "Relação com o saber" e suas possibilidades para a análise psicopedagógica das aprendizagens escolares". Psicopedagogia, 31(96), 275-288. Recuperado de http://pepsic.bvsalud.org/pdf/psicoped/v31n96/05.pdf 2014.

VERCELLINO, Soledad. La escuela y los (des)encuentros con el saber. Viedma: Editorial UNRN. 2018.

ZAMBRANO, Armando. Relación con el saber, fracaso/éxito escolar y estrategias de enseñanza-aprendizaje. Actualidades Pedagógicas, 61, 27-43. doi: https://doi.org/10.19052/ap.2329. 2013. 


\section{7 usw \\ ...

ISSN: 1984-6444 | http://dx.doi.org/10.5902/1984644448156

\section{Correspondência}

Soledad Vercellino - Universidad Nacional de Río Negro - Centro Interdisciplinario de Estudios sobre Derechos, Inclusión y Sociedad. Río Negro, Argentina

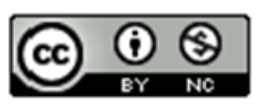

This work is licensed under a Creative Commons Attribution-NonCommercial 4.0 International (CC BY-NC 4.0)

\section{Notas}

\footnotetext{
${ }^{1}$ La autora agradece a la Universidad Nacional de Río Negro y a la Universidad Nacional del Comahue que han financiado los proyectos de investigación en el marco de los cuales surge esta comunicación.

${ }^{2}$ Que amplía y actualiza las revisiones ya realizadas en: VERCELLINO 2015B, 2018 Y VERCELLINO, VAN DEN HEUVEL Y GUERREIRO, 2014
} 